\title{
Secuencia de perfusión arterial reversa en embarazo gemelar; coagulación de arteria nutricia. Reporte del primer caso en el país
}

Twin reversed arterial perfusion sequence; coagulation of the umbilical cord. A report on the first case in Uruguay

Seqüência reversa de perfusão arterial na gravidez gemelar; coagulação da artéria nutritiva. Relato do primeiro caso no país

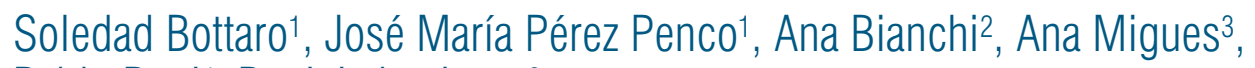
Pablo Putti ${ }^{4}$, Patricia Lapizaga3 ${ }^{3}$

\section{Resumen}

La secuencia de perfusión arterial reversa (TRAP) es una complicación muy poco frecuente y grave de los embarazos gemelares monocoriónicos. Generalmente ocurre cuando el corazón de un gemelo de apariencia normal sirve como bomba para uno o más gemelos dismórficos cuya cabeza, órganos torácicos y extremidades superiores no se desarrollan completamente o no se desarrollan en absoluto y, por lo tanto, carecen de actividad cardíaca. La arquitectura placentaria vascular anómala provoca un cambio en el flujo arterial hacia el gemelo acardíaco. Los mecanismos fisiopatológicos exactos que conducen a este fenómeno devastador no se conocen bien. Compartiremos el caso clínico de una paciente de 19 años, cursando un embarazo gemelar monocorial monoamniótico, en que realizamos diagnóstico de TRAPS, y realizamos la coagulación laser de la arteria nutricia del feto acárdico.

Palabras clave: Gemelo acárdico

Embarazo gemelar

Gemelos monocoriónicos

Secuencia de perfusión arterial reversa

Secuencia TRAP

Key words: $\quad$ Acardiac twin

Pregnancy, twin

Monochorionic twins

Reverse arterial perfusion sequence

TRAP sequence

\footnotetext{
1. Unidad de Medicina Prenatal. Hospital Pereira Rossell. Docente Asociado de la Clínica Ginecotocológica "A". Facultad de Medicina. UDELAR. 2. Jefa de la Unidad de Medicina Prenatal. Hospital Pereira Rossell.

3. Unidad de Medicina Prenatal. Hospital Pereira Rossell. ASSE

4. Prof. Adj. de la Clínica Ginecotocológica "A"' Facultad de Medicina. UDELAR.

Los autores declaran no tener conflicto de intereses.

Se solicitó consentimiento informado a la paciente para su publicación.

Correspondencia: Soledad Bottaro. Correo electrónico: solebottaro@gmail.com

Fecha de recibido 19/2/2021

Fecha de aprobado 28/4/2021

Attribution-NonCommercial 4.0 International (CC BY-NC 4.0)
} 


\section{Introducción}

La secuencia de perfusión arterial reversa (TRAP) es una complicación rara, exclusiva de los embarazos múltiples monocoriales. Su incidencia se calcula en 1:35.000 nacimientos y representa el $1 \%$ de los gemelos monocoriales ${ }^{(1)}$. Se produce en las dos terceras partes de los casos en gemelares monocoriales biamnióticos y un tercio en monocoriales monoamnióticos ${ }^{(2)}$.

Es una patología malformativa y vascular grave de los embarazos monocoriales. Esta se compone de dos fetos, uno sin alteraciones y el otro con malformaciones graves, cuya alteración primaria es la ausencia del corazón. A su vez la placenta presenta anastomosis arterio-arteriales (AA) y veno-venosas (VV) superficiales, que genera el paso de sangre del feto normal (denominado bomba) al feto malformado (denominado acárdico), por lo que este último constituye un verdadero parásito para el gemelo sano.

En la secuencia TRAP la sangre (pobremente oxigenada) fluye del gemelo sano (donor o pump twin) en dirección inversa hacia la arteria umbilical del gemelo acárdico a través de una anastomosis $\mathrm{AA}^{(3,4)}$. La vena umbilical del feto parásito retorna la sangre a la placenta y de aquí al gemelo donante, con el consecuente riesgo de anemia, fallo circulatorio y muerte.

La presencia de estas anastomosis AA permite el flujo reverso y es una condición imprescindible para definir este cuadro ${ }^{(5,6)}$.

Invariablemente el feto acárdico presenta un cordón umbilical con dos vasos. Además los sistemas circulatorios y linfáticos no están comunicados en la zona superficial, lo que genera un edema subcutáneo e higromas quísticos que hacen más difícil su diferenciación.

A medida que crece el gemelo acardíaco, aumenta la demanda de sangre del feto con bomba. Por lo tanto, pueden ocurrir insuficiencia cardíaca de alto gasto, hidropesía fetal y polihidramnios en el gemelo bomba. Si la secuencia TRAP no se trata, la tasa de mortalidad del feto con bomba es aproximadamente del $55 \%{ }^{(7)}$. Después de un diagnóstico de secuencia TRAP, un tercio de los fetos con bomba muere antes de las 16 semanas de gestación ${ }^{(8)}$.

Son criterios de mal pronóstico el deterioro cardiovascular, representados por polihidramnios, cardiomegalia, derrame pleural, regurgitación tricuspídea, onda "a" reversa en el ductus venoso y pulsatilidad de la vena umbilical. El aumento de la velocidad máxima en la arteria cerebral media (ACM) es un signo de anemia fetal.

Presentamos a continuación el caso clínico de una paciente del Centro Hospitalario Pereira Rossell, cursando un embarazo gemelar complicado con una secuencia TRAP y en que se pudo ofrecer finalmente un

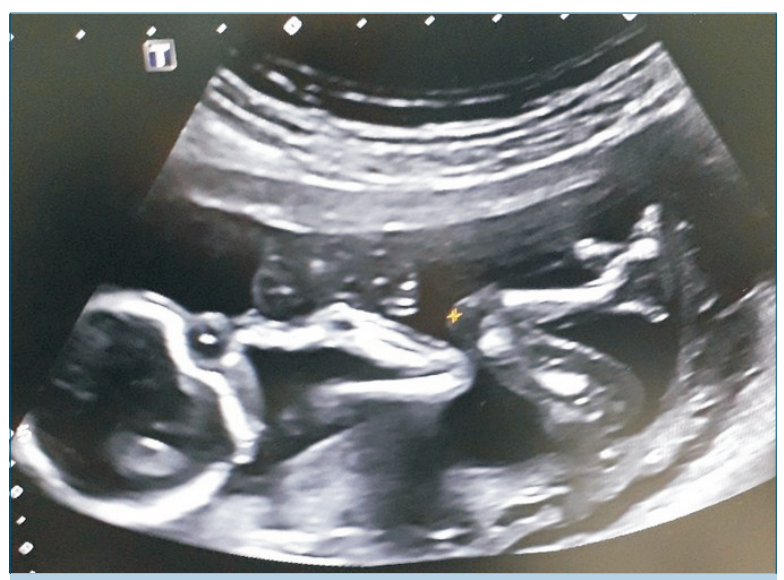

Figura 1. Gemelo de 13 semanas sano.

tratamiento fetal realizado por primera vez en nuestro medio.

\section{Caso clínico}

Paciente de 19 años, sana, con antecedente de una gestación previa que se interrumpe por agenesia renal bilateral. Embarazo buscado, de captación a las 9 semanas. En screening del primer trimestre del embarazo, a las 13 \pm 3 semanas, se identifica un feto vivo de $70,7 \mathrm{~mm}$ de longitud céfalo-caudal, con marcadores ecográficos de cromosomopatías (translucencia nucal, hueso nasal, ductus venoso y flujo tricuspídeo) normales y un segundo feto, sin actividad cardíaca en proceso de reabsorción.

La paciente es derivada a nuestro centro, la Unidad de Medicina fetal (UMP) del Centro Hospitalario Pereira Rossell, para revalorar. Cursando un embarazo de 15 \pm 6 semanas, realizamos diagnóstico de embarazo monocorial monoamniótico, identificándose un feto vivo y sano, con crecimiento acorde para la edad gestacional (figura 1) y conjuntamente una masa amorfa sin actividad cardíaca (figura 2), con edema subcutáneo generalizado de 35 × 20 × $28 \mathrm{~mm}$ (dimensiones aumentadas con respecto a un estudio previo) por lo que se plantea diagnóstico de embarazo gemelar con feto hidrópico en un caso de secuencia TRAP.

Dado que no contamos en nuestro en nuestro país con el equipamiento necesario para el tratamiento de esta complicación, en un feto hemodinámicamente estable y ante la situación actual de pandemia por COVID-19 se realiza seguimiento semanal con ecografía y Doppler fetoplacentario.

De la ultrasonografía realizada a las $17 \pm 6$ semanas, se destaca feto sano con movimientos y actividad cardíaca fetal presente, y crecimiento fetal adecuado para la edad gestacional. La anatomía fetal es normal para la 

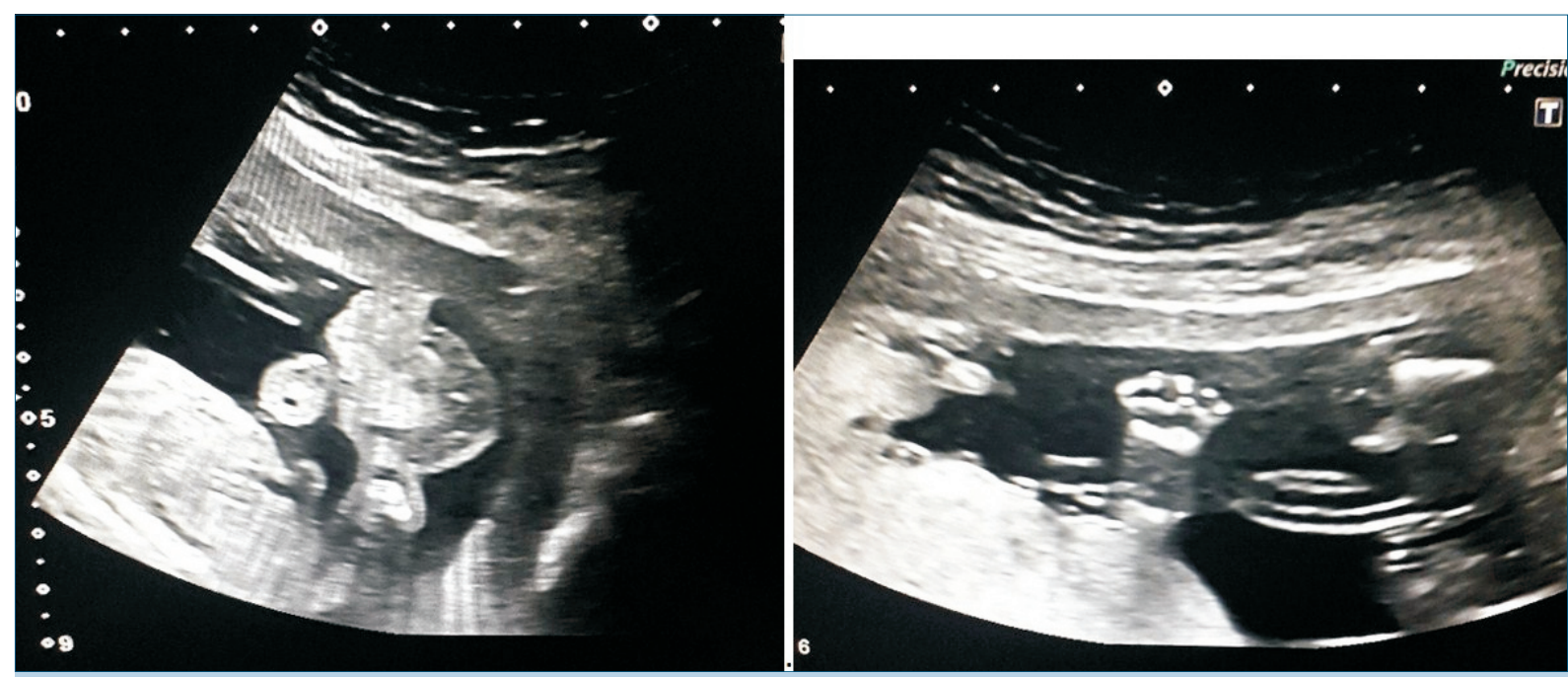

Figura 2. En la imagen izquierda se visualiza masa amorfa con edema generalizado a su alrededor. En imagen derecha, parte de la masa amorfa donde se identifica un miembro inferior. No se identifica cráneo ni miembros superiores.

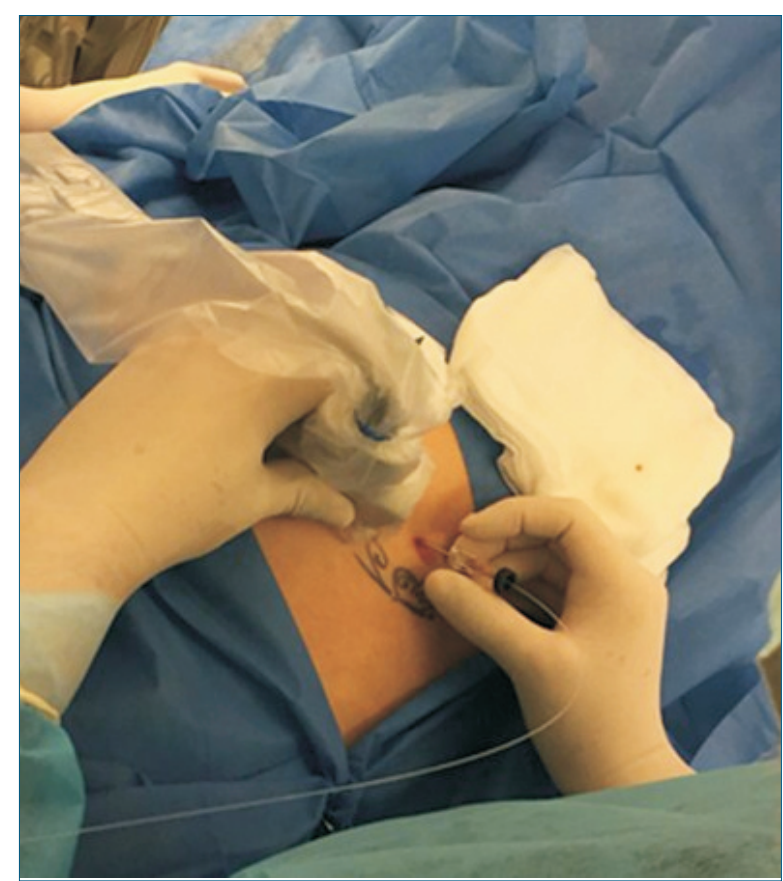

Figura 3. Cirugía láser.

edad gestacional. El feto se presentaba con buen estado hemodinámico fetal y sin anemia. La masa amorfa en esta segunda instancia había aumentado de tamaño.

Con la colaboración y adecuada coordinación se consiguió un equipo láser para realizar el tratamiento a nuestra paciente. Se realiza coagulación láser de la arteria nutricia del feto hidrópico cursando $18 \pm 4$ semanas.

El procedimiento fue llevado a cabo en block quirúrgico con ligera sedación de la paciente (figura 3). No hubo complicaciones secundarias al mismo y fue bien tolerado por la paciente. Se comprueba la vitalidad del feto sano a la hora del procedimiento. Dada la buena evolución, la paciente se presentó asintomática a las 24 horas, con ecografía se seguimiento normal y Doppler normal en el feto sano, se otorgó alta a domicilio.

Se realizó seguimiento a la semana del procedimiento, cursando $19 \pm 1$ semanas. El crecimiento del feto sano es adecuado para la edad gestacional, no se comprueba anemia fetal y el estado hemodinámico fetal es normal.

Continúa controles obstétricos y ecográficos en nuestra unidad (UMP). Cursa embarazo asintomática y sin complicaciones. Ingresa cursando 37 semanas con diagnóstico de rotura prematura de membranas. Se induce trabajo de parto, dado patrón no alentador de la frecuencia cardíaca fetal se realiza operación cesárea. Se obtiene un recién nacido vivo, de sexo masculino de 2540 gramos y APGAR 9/10. Se adjunta imagen de placenta y masa amorfa (figura 4).

Pasan a alojamiento conjunto. Se otorga alta a las 72 horas dada la buena evolución del binomio madre-hijo.

\section{Discusión}

En la última década, la mejora de las técnicas ecográficas ha permitido el diagnóstico de la secuencia TRAP en el primer trimestre.

Se debe considerar el diagnóstico prenatal de un gemelo acardíaco cuando en el examen ecográfico se 


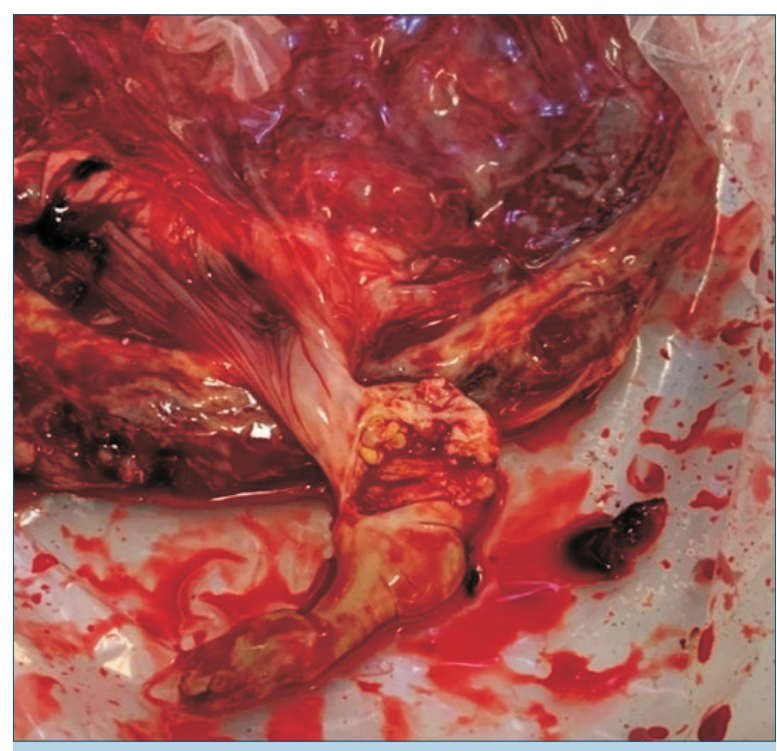

Figura 4. Placenta con masa amorfa. Se puede apreciar una imagen que corresponde a un miembro inferior, como se visualizaba en la ultrasonografía.

muestra un embarazo gemelar monocoriónico y uno de los fetos presenta anomalías morfológicas evidentes ${ }^{(9)}$.

Los principales objetivos en el manejo de la secuencia TRAP son preservar la supervivencia del gemelo bomba y alcanzar el plazo para el parto. Para esto existen diferentes métodos terapéuticos.

Aún se debate el momento óptimo de la intervención terapéutica. El tratamiento con TRAP generalmente se realiza entre las 16 y 18 semanas, con la hipótesis de que el riesgo de aborto espontáneo podría ser menor después de la obliteración espontánea de la cavidad celómica. Sin embargo, la tasa de pérdida espontánea de embarazos TRAP reportada en la literatura está entre $35 \%$ y $50 \%{ }^{(9,10)}$.

El abordaje intrafetal tiene como objetivo la ablación de los vasos pélvicos o la aorta abdominal del gemelo acardíaco, que se pueden identificar fácilmente en la ecografía Doppler color. Este procedimiento no se ve afectado por la ubicación de la placenta, la estructura del cordón umbilical, el volumen de líquido amniótico y la posición del acardio.

El único tratamiento causal de la secuencia TRAP es la interrupción del flujo sanguíneo al gemelo acardíaco. Se han descrito varias técnicas fetoscópicas, incluida la coagulación del cordón, la ligadura del cordón y la fotocoagulación de las anastomosis, así como métodos intrafetales como la ablación por radiofrecuencia y la terapia con láser intrafetal ${ }^{(11-14)}$.

Pagani y colaboradores ${ }^{(15)}$, en un estudio de cohorte de 23 casos, 17 tratados con intervención y 6 tratados de forma conservadora, informaron una alta tasa de pérdida espontánea inesperada en el grupo de tratamiento conservador (83\%). Los datos de los casos tratados en este estudio se combinaron luego con los datos de otros diez estudios, y el análisis mostró un resultado adverso del embarazo significativamente menor en el grupo en el que se realizó el tratamiento antes de las 16 semanas.

La intervención temprana en embarazos con secuencia TRAP se hizo posible con la introducción de la ablación laser intrafetal guiada por ultrasonido de la perfusión del gemelo acárdico ${ }^{(16)}$.

\section{Conclusiones}

El manejo y el tratamiento de la secuencia TRAP plantean desafíos sin resolver, ya que aún deben aclararse tanto la técnica elegida como el momento óptimo de intervención. Si bien la literatura actual es rica en estudios, su alta heterogeneidad hace que las comparaciones sean propensas al sesgo. Si se necesita tratamiento, todavía se debate el mejor momento para la intervención, aunque los últimos estudios fomentan la intervención en el primer trimestre del embarazo. En cuanto a la técnica de elección para interrumpir el suministro vascular al gemelo acardíaco, la coagulación con láser guiada por ultrasonido y la ablación por radiofrecuencia de los vasos intrafetales suelen ser los enfoques preferidos.

\section{Abstract}

Twin reversed arterial perfusion sequence (TRAPS) is rather an unusual and severe complication of monochorionic twin pregnancies. It usually occurs when the normal-appearance heart of a twin acts as a pump for one or more dysmorphic twins whose head, thoracic organs and upper limbs fail to totally develop or do not develop at all and thus, have no cardiac activity. The abnormal vascular architecture at the placenta changes the arterial flow towards the acardiac twin. The exact pathophysiological mechanisms that result in this devastating phenomenon are still unknown. The study presents the clinical case of a 19-year- old patient pregnant with monoamniotic, monochorionic twins and a diagnosis of TRAPS, treated by laser coagulation of the acardiac twin's umbilical cord.

\section{Resumo}

A seqüência reversa de perfusão arterial (TRAPS) é uma complicação muito rara e grave de gestações gemelares monocoriônicas. Geralmente ocorre quando o coração de um gêmeo de aparência normal serve como uma bomba para um ou mais gêmeos dismórficos cuja cabeça, órgãos torácicos e membros superiores não se desenvolvem totalmente ou não se desenvolvem e, portanto, não têm atividade cardíaca. A arquitetura vascu- 
lar placentária anormal causa uma mudança no fluxo arterial para o gêmeo acardíaco. Os mecanismos fisiopatológicos exatos que levam a esse fenômeno devastador não são bem compreendidos. Descrevemos o caso clínico de uma paciente de 19 anos, portadora de gestação gemelar monocoriônica monoamniótica, na qual fizemos o diagnóstico de TRAPS e realizamos coagulação a laser da artéria nutritiva do feto acardíaco.

\section{Bibliografía}

1. van Gemert MJ, Ross MG, Nikkels PG, Wijngaard JP. Acardiac twin pregnancies part III: model simulations. Birth Defects Res A Clin Mol Teratol 2016; 106(12):1008-15.

2. Benirschke K, Kaufmann P, Baergen R. Multiple pregnancy. En: Benirschke K, Kaufmann P, Baergen R. Pathology of the human placenta. 2nd ed. New York, NY: Springer, 1995:702-10.

3. Steffensen TS, Gilbert-Barness E, Spellacy W, Quintero RA. Placental pathology in trap sequence: clinical and pathogenetic implications. Fetal Pediatr Pathol 2008; 27(1):13-29.

4. Deprest J, Gratacós E. Embarazos monocoriales (II): gemelos discordantes. Indicaciones y técnicas de oclusión de cordón umbilical. En: Gratacós E, Gómez R, Nicolaides K, Romero R, Cabero L. Medicina Fetal. Madrid: Médica Panamericana, 2007:703-8.

5. Chalouhi GE, Stirnemann JJ, Salomon LJ, Essaoui M, Quibel T, Ville Y. Specific complications of monochorionic twin pregnancies: twin-twin transfusion syndrome and twin reversed arterial perfusion sequence. Sem Fetal Neonatal Med 2010; 15(6):349-56.

6. Hecher K, Lewi L, Gratacos E, Huber E, Ville Y, Deprest $\mathbf{J}$. Twin reversed arterial perfusion: fetoscopic laser coagulation of placental anastomoses or the umbilical cord. Ultrasound Obstet Gynecol 2006; 28:688-91.

7. Moore TR, Gale S, Benirschke K. Perinatal outcome of forty-nine pregnancies complicated by acardiac twinning. Am J Obstet Gynecol 1990; 163:907-12.
8. Lewi L, Valencia C, Gonzalez E, Deprest J, Nicolaides KH. The outcome of twin reversed arterial perfusion sequence diagnosed in the first trimester. Am J Obstet Gynecol 2010; 203:213.e1-4.

9. Sepúlveda WH, Quiroz VH, Giuliano A, Henríquez R. Prenatal ultrasonographic diagnosis of acardiac twin. J Perinat Med 1993; 21(3):241-6.

10. van Gemert MJ, Umur A, van den Wijngaard JP, VanBavel E, Vandenbussche FP, Nikkels PG. Increasing cardiac output and decreasing oxygenation sequence in pump twins of acardiac twin pregnancies. Phys Med Biol 2005; 50(3):N33-42.

11. Jolly M, Taylor M, Rose G, Govender L, Fisk NM. Interstitial laser: a new surgical technique for twin reversed arterial perfusion sequence in early pregnancy. BJOG 2001; 108(10):1098-102.

12. Tan TY, Sepulveda W. Acardiac twin: a systematic review of minimally invasive treatment modalities. Ultrasound Obstet Gynecol 2003; 22(4):409-19.

13. Hecher K, Lewi L, Gratacos E, Huber A, Ville Y, Deprest J. Twin reversed arterial perfusion: fetoscopic laser coagulation of placental anastomoses or the umbilical cord. Ultrasound Obstet Gynecol 2006; 28(5):688-91.

14. Livingston JC, Lim FY, Polzin W, Mason J, Crombleholme TM. Intrafetal radiofrequency ablation for twin reversed arterial perfusion (TRAP): a single ] center experience. Am J Obstet Gynecol 2007; 197(4):399.e1-3.

15. Pagani G, D'Antonio F, Khalil A, Papageorghiou A, Bhide A, Thilaganathan B. Intrafetal laser treatment for twin reversed arterial perfusion sequence: cohort study and meta-analysis. Ultrasound Obstet Gynecol 2013; 42:6-14.

16. Jolly M, Taylor M, Rose G, Govender L, Fisk NM. Interstitial laser: a new surgical technique for twin reversed arterial perfusion sequence in early pregnancy. BJOG 2001; 108(10):1098-102.

\footnotetext{
Contribución de autores

Soledad Bottaro, ORCID 0000-0003-2550-9148. Concepción, diseño, análisis y redacción. José María Pérez Penco, ORCID 0000-0002-3665-7851. Concepción y análisis.

Ana Bianchi, ORCID 0000-0003-1045-1190. Revisión crítica.

Ana Migues, ORCID 0000-0003-4429-5093. Análisis y redacción.

Pablo Putti, ORCID 0000-0002-6566-8242. Análisis y revisión crítica.

Patricia Lapizaga, ORCID 0000-0001-5748-7671. Diseño y redacción.
} 